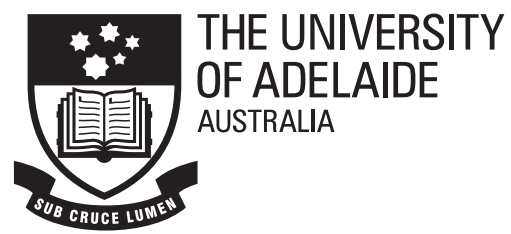

Faculty of Engineering, Computer and Mathematical Sciences SCHOOL OF MECHANICAL ENGINEERING

\title{
Ocean acoustic interferometry
}

DOCTORAL THESIS

$12^{\text {th }}$ October 2008

\section{Author: Laura A. Brooks}

\author{
Supervisor: $\quad$ Assoc. Prof. Anthony C. Zander ${ }^{\dagger}$ \\ Co-supervisors: Dr Peter Gerstoft ${ }^{\dagger \dagger}$ \\ Prof. Colin H. Hansen ${ }^{\dagger}$ \\ Dr Z. Yong Zhang *
}

\footnotetext{
${ }^{\dagger}$ School of Mechanical Engineering, The University of Adelaide, Australia

${ }^{\dagger \dagger}$ Marine Physical Laboratory, Scripps Institution of Oceanography, USA

*Defence Science and Technology Organisation, Edinburgh, Australia
} 


\section{Chapter 5}

\section{Ship Dominated Ambient Noise Cross-correlation}

The high sea states and wind conditions created by Tropical Storm Ernesto on September 22006 created an opportunity to explore underwater noise fields with unusual characteristics. Within this chapter cross-correlations of ship dominated $20-100 \mathrm{~Hz}$ noise collected on three L-shaped arrays from August 31 to September 32006 are considered in detail. In particular, the applicability of common time and frequency domain preprocessing techniques to ship dominated ambient noise cross-correlation, the use of various receiver geometric configurations, and the effect of temporal variations on the cross-correlations, are all investigated. Knowledge of these is necessary in order not just to obtain good approximations to the Green's function from cross-correlation of ship dominated ambient noise, but also to understand the limitations of these approximations. The data were collected using the equipment at the locations described in Chapter 4.

A significant proportion of the work in this chapter has been submitted for publication in JASA [26]. 


\subsection{Introduction}

Although ocean acoustic interferometry (OAI) theory prescribes a uniform noise distribution, good approximations of the arrival structure of the actual Green's function can still be obtained from the cross-correlation timederivative, termed the empirical Green's function approximation (EGFA), even when the distribution is not completely uniform, as demonstrated in the seismic literature $[11,12,80]$. At any time the cross-correlation of the data analysed from the experiments here tends to be dominated by one or two nearby ships, and therefore in order to obtain a cross-correlation that does not have directional bias, the time period over which the crosscorrelation is performed must be sufficiently long such that several ship tracks that pass through the end-fire region are included [25]. When this is not the case spurious precursory arrivals corresponding to cross-correlations between paths from directions that are more densely sampled are apparent in the summed cross-correlation.

During Tropical Storm Ernesto most ships in the vicinity of the experimental area left the region. Thus, the shipping noise field was dominated by distant vessels. The reduced local shipping traffic, along with elevated wind and sea state conditions, meant that a greater amount of acoustic energy in the ocean at low frequencies was from both local and distant breaking waves, and distant shipping, than at other times. The overall acoustic energy levels were also higher. Because of the higher signal levels and the more uniform directivity of the shipping noise field, the arrival-time structure of EGFAs for low frequency cross-correlations of data recorded during the storm should more closely match that of the actual Green's functions between the hydrophones than during calmer periods.

The temporal characteristics of the ocean are non-stationary. This suggests that cross-correlations over short time periods, such as a few minutes, are optimal if instantaneous EGFAs are desired. However, the need to average over multiple ship tracks requires longer cross-correlation times, and hence cross-correlations that represent the 'average' EGFA over long time 
periods of 24 hours are determined here. The theory assumes that sources all have the same amplitude and frequency content. Nearby ships tend to be louder, and larger ships have spectra that are dominated by lower frequencies. Time and frequency preprocessing are carried out to minimise these effects. The theory also assumes that ships move along a regular path at constant velocity. Ships that change velocity or have sharp changes in direction will degrade the EGFA. A ship that remains stationary in one location for a long time period is shown to result in a spurious peak at the corresponding time.

Cross-correlations between different sets of hydrophones within each array are compared, and it is shown that the cross-correlation yields direct and surface reflected paths between horizontal line array (HLA) hydrophones, and that bottom-surface reflected paths between HLA and vertical line array (VLA) hydrophones can be determined. Due to the long wavelengths considered, no attempt is made to determine sediment paths. Crosscorrelations for equi-spaced hydrophones in an HLA are shown to have relatively little variation in amplitude and frequency, particularly at the crosscorrelation peaks. Summing the cross-correlations between equally spaced pairs is shown to increase the signal-to-noise ratio (SNR). Temporal characteristics of the cross-correlations are also examined, and cross-correlations from calm periods are compared to those obtained from data collected during the tropical storm.

\subsection{Theory}

At frequencies above a few hundred Hertz the ocean sound field is dominated by surface noises from ocean waves [22-24]. At frequencies below about $100 \mathrm{~Hz}$ the noise field is dominated by shipping noise [22, 23]. Nearby shipping favours higher grazing angles between the acoustic paths and the horizontal, while distant shipping favours more horizontally travelling wavefronts. Noise in the frequency range $20-100 \mathrm{~Hz}$ is considered here. If it is 
assumed that ship noise can be modelled as a set of sources that are uniformly and densely distributed within a horizontal plane near the surface of a waveguide, then the cross-correlation between two receivers can be derived following the stationary phase methodologies of Section 3.1 and Refs. [30] and [69]. The cross-correlation of the signals recorded at two receivers, $A$ and $B$, is, from Eq. (3.4):

$$
C_{A B}(\omega)=|\rho S(\omega)|^{2} n \iint G\left(\mathbf{r}_{A}, \mathbf{r}_{S}\right) G^{*}\left(\mathbf{r}_{B}, \mathbf{r}_{S}\right) d x d y,
$$

where $S(\omega)$ is the ship source spectrum, $\rho$ is the density of the medium, $n$ is the number of sources per unit area, $G\left(\mathbf{r}_{\psi}, \mathbf{r}_{S}\right)$ is the Green's function between the source, $S$, and receiver, $\psi,{ }^{*}$ denotes the complex conjugate, and $x$ and $y$ are the horizontal axes parallel and perpendicular to the vertical plane containing $A$ and $B$ respectively.

The full Green's function at each receiver can be written as the superposition of direct and reflected waves. For a uniform sound speed waveguide, bounded by a free surface and a bottom with reflection coefficient $\Gamma$, the Green's function between the source, $S$, and receiver, $\psi$, can be expressed as a sum of free-field Green's functions in the same manner as for Eq. (3.6) [1, 69]:

$$
\begin{aligned}
G\left(\mathbf{r}_{\psi}, \mathbf{r}_{S}\right)= & \sum_{b_{\psi}=0}^{\infty} \Gamma^{b_{\psi}} G_{f}\left(\sqrt{\left(x-x_{\psi}\right)^{2}+y^{2}+\left(2 b_{\psi} D+z \pm z_{\psi}\right)^{2}}\right) \\
& +\sum_{b_{\psi}=1}^{\infty} \Gamma^{b_{\psi}} G_{f}\left(\sqrt{\left(x-x_{\psi}\right)^{2}+y^{2}+\left(2 b_{\psi} D-z \pm z_{\psi}\right)^{2}}\right),
\end{aligned}
$$

where $b_{\psi}$ is the number of bottom bounces for a given path, $D$ is the depth of the waveguide, the $y=0$ horizontal axis is defined as that which contains both $A$ and $B$, and $G_{f}(R)=\frac{e^{i k R}}{4 \pi R}$ is the 3D Green's function within a homogeneous medium, where $k$ is the wave number and $R$ is the total distance that a particular wave travels. The first term on the RHS of Eq. (5.2) includes all up-going waves, and the second term includes all downgoing waves as measured from the source.

Inserting Eq. (5.2) into Eq. (5.1) yields a cross-correlation expression that consists of the sum of the integrals of all possible combinations of 
the interaction between any path to the first receiver, and any path to the second. Consider any of these individual interactions. Substitution of Eq. (5.2) into Eq. (5.1), that is, cross-correlation between two arbitrary paths, yields $[30,69]$

$$
C_{A B}(\omega)=|\rho S(\omega)|^{2} n \frac{\Gamma^{b_{A}+b_{B}}}{(4 \pi)^{2}} \iint \frac{e^{i k\left(L_{A}-L_{B}\right)}}{L_{A} L_{B}} d x d y
$$

where $b_{\psi}$ is the number of bottom bounces for the path to $\psi$, and

$$
L_{\psi}=\sqrt{\left(x-x_{\psi}\right)^{2}+y^{2}+\left(2 b_{\psi} D \pm z \pm z_{\psi}\right)^{2}}
$$

is the length of the given path between the source, $S$, and receiver, $\psi$.

Application of the method of stationary phase to Eq. (5.3) [15, 30, 68, 69], and summation over all stationary points, yields

$$
C_{A B}(\omega)=i n|S(\omega)|^{2} \sum_{\chi_{s}}\left(\frac{\Gamma^{b_{A}+b_{B}} c \rho}{2 \omega \cos \theta} G_{f}\left(R\left(\chi_{s}\right)\right)\right),
$$

where $c$ is the wave velocity, $f$ is the acoustic frequency, $\omega=2 \pi f$ is the angular frequency, $\theta$ is the acute angle between the ray path and the vertical, and $\chi_{s}$ are the stationary points. Note that the stationary points satisfy the relationship $\theta_{A}= \pm \theta_{B}$. The positive relationship between $\theta_{A}$ and $\theta_{B}$ only occurs when the path to the furthest receiver passes through the closer receiver, hence the relationship between the summed cross-correlations and the Green's function between the receivers. The negative relationship corresponds to stationary-phase contributions from cross-correlations between a wave that initially undergoes a surface reflection, and one that does not $[15,69]$. Since ship sources are near the ocean surface, these spurious arrivals will converge to almost the same time delay as the true Green's function paths, and due to the long wavelengths, will not be observed as separate peaks. The theory presented here has neglected curvature of ray paths due to refraction, but it has been shown by others [30] that the stationary phase argument generalises to a heterogenous medium with smooth velocity variations. 
The cross-correlation in Eq. (5.5) can therefore be seen to produce an amplitude and phase shaded Green's function. The amplitude shading is dependent on the travel path through the $\Gamma^{b_{A}+b_{B}}$ and $\cos \theta$ terms, and also contains both constant and frequency dependent components. The $1 / \omega$ factor phase shading in Eq. (5.5) means that the time domain Green's function is proportional to the derivative of the summed cross-correlations [11, 13 , 15, 69]:

$$
\frac{\partial C_{A B}(t)}{\partial t} \simeq-\left[G_{A B}(t)-G_{A B}(-t)\right] .
$$

The raw cross-correlation, rather than its time derivative, is often used as an approximation to the Green's function $[25,56,65,66]$, and for a midhigh frequency finite bandwidth signal this can be a good approximation since the cross-correlation peaks at roughly the same time as its derivative.

\subsection{Experiment}

Data considered in this chapter were collected from August 31 through September 3 on the three L-shaped arrays: SWAMI52, SWAMI32, and Shark. Array locations and orientations are shown in Figure 5.1(a), and configurations are detailed in Table 5.1. The HLAs were all located on the seafloor. All VLA hydrophones and the Shark HLA hydrophones are evenly spaced. SWAMI52 inter-hydrophone distances increase from the centre, and SWAMI32 HLA inter-hydrophone distances increase from the hydrophone 13 (H-13) end respectively. The exact HLA hydrophone spacing of each SWAMI array is detailed in Appendix B.1.2. A sketch of the SWAMI52 array geometry is shown in Figure 5.1(b). SSPs that were recorded near SWAMI52 on August 30 and September 6 are shown in Figure 5.1(c).

Tropical Storm Ernesto created large sea states and high winds. The wind direction and speed from August 31 through September 3 are shown in Figure 5.1(d)-(e). Predominantly easterly winds gradually built up over August 31 and September 1 to a $20 \mathrm{~m} / \mathrm{s}$ peak early on September 2, and then remained high until late in the day, when they dropped rapidly once 
(a)

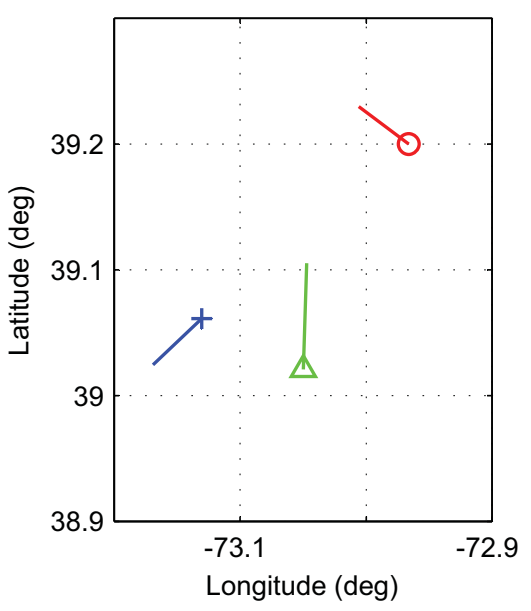

(b)

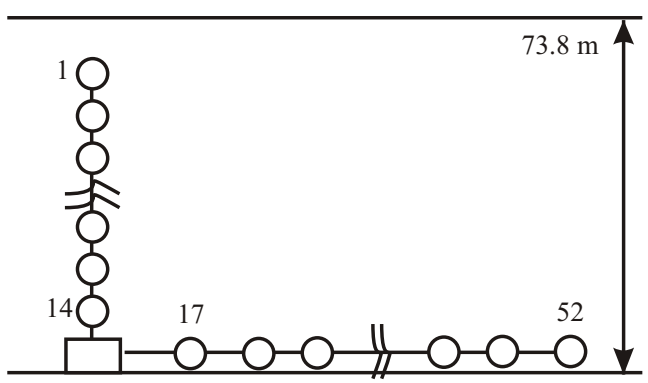

(c)

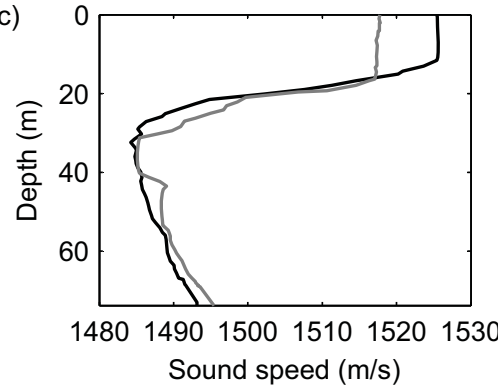

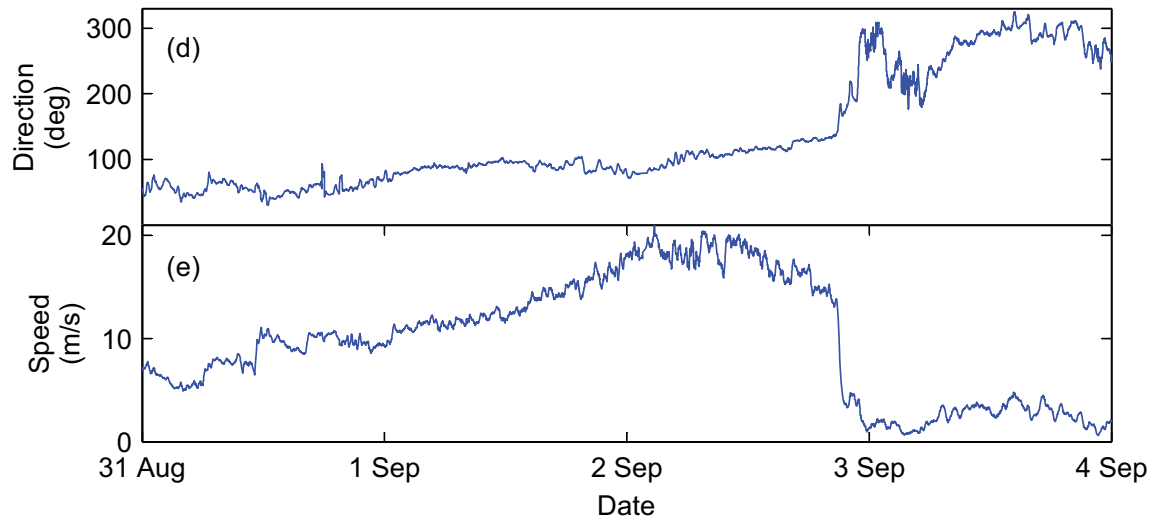

Figure 5.1: (a) The relative VLA locations of SWAMI52 (O), SWAMI32 $(+)$, and Shark $(\triangle)$. The lines departing each VLA show the HLA orientation. The array length is scaled by a factor of 20. (b) SWAMI52 array geometry and hydrophone numbering system. (c) SSPs near SWAMI52 for August 30 (black) and September 6 (grey). (d) Wind direction and (e) wind speed, from R/V Knorr ship records, from August 31 to end of September 3. 
Table 5.1: Details of array configurations. ${ }^{a}$ Lowest numbered hydrophone is uppermost in the array. Extra hydrophones tied off just above frame (SWAMI52: H-15 and H-16, SWAMI32: H-11 and H-12, Shark: H-13-H15). ${ }^{b}$ Lowest numbered hydrophone is closest to array except for Shark, which is opposite. ${ }^{c}$ Data from H-15 and H-46 were discarded due to inconsistencies with other data.

\begin{tabular}{ccccccc}
\hline \hline & \multirow{2}{*}{$\begin{array}{c}\text { Water depth } \\
(\mathrm{m})\end{array}$} & $\begin{array}{c}\text { Number of } \\
\text { hydrophones }\end{array}$ & Length $(\mathrm{m})$ & Hydrophones $^{\mathrm{a}}$ & Length $(\mathrm{m})$ & Hydrophones $^{\mathrm{b}}$ \\
\cline { 5 - 7 } & & 52 & 56.81 & $1: 14$ & 230 & $17: 52$ \\
SWAMI52 & 73.8 & 32 & 53.55 & $1: 10$ & 256 & $13: 32$ \\
SWAMI32 & 68.5 & 48 & 64.25 & $0: 12$ & 465 & $16: 47^{\mathrm{c}}$ \\
Shark & 79 & 48 & & & &
\end{tabular}

the storm had passed. The decrease in speed was accompanied by a change in wind direction to south and west.

On September 2 several of the SWAMI32 channels switched, as described in detail in Chapter 7. Corrections for this were applied to the relevant data presented here.

\subsection{Analysis of data preprocessing methods}

Time and frequency domain preprocessing methods were applied to the raw data to emphasise broadband ocean noise. The preprocessing techniques considered here were analysed using data collected on SWAMI52 throughout September 2 (Zulu time). No towed source experiments were undertaken on this day, because of Ernesto, and therefore ocean noise over a large frequency bandwidth could be considered. The data were stored and analysed in 140 portions, each 10 minutes and 14 seconds duration, or 10:14 min.

Short (10:14 min) cross-correlations were unstable at shallow depths above the thermocline, likely due to sound speed fluctuations resulting from elevated levels of swell and mixing due to Ernesto. If the noise field had been isotropic and sufficiently strong, cross-correlations could have been performed over periods that were sufficiently short for the environment to 
Analysis of data preprocessing methods

be considered stationary. The temporal change in cross-correlation could then have been related to environmental changes, in particular changes in temperature in the upper waveguide, and tidal changes. However, the dominant noise here was from discrete ships, and therefore the cross-correlations had to be performed over a long time period so that specific sources did not dominate (see Section 5.6). Cross-correlations were therefore performed over the entire day. Direct path propagation between hydrophones lower in the water column would not have changed significantly over this time, but any propagation paths that passed through the thermocline region may have. The resulting EGFA is therefore an approximation of the 'average' Green's function over the day.

\subsubsection{Removal of main contamination}

Depending upon the particular time interval, some of the September 2 data exhibited one or more of the following: high amplitude mid-frequency signal from fixed location sound sources, amplitude clipping, and low frequency energy bursts. Spectrograms and time series of data collected on September 2, showing examples of each of these aberrations, are shown in Figure 5.2.

Three fixed location sound source signals are observed in Figure 5.2(a). A $300 \mathrm{~Hz}$ 1.5-second duration LFM (linear frequency modulated) signal with a $60 \mathrm{~Hz}$ bandwidth is visible in the spectrogram as ramps that cut on and off at regular intervals. The LFM signal is bounded above and below by two continuous horizontal lines of high amplitude, created by phase encoded signals at $224 \mathrm{~Hz}$ and $400 \mathrm{~Hz}$, both with $16 \mathrm{~Hz}$ bandwidth. The amplitude of the time-series is seen to spike when the LFM signal is active.

An example of amplitude clipping is shown in Figure 5.2(b). The frequency of the signal that exceeds the maximum allowable amplitude is low, and since the spectrogram is normalised to the maximum value at any given time, the apparent amplitude of all higher frequencies is reduced, yielding vertical green and yellow lines in the spectrogram. A low frequency energy burst is visible in Figure 5.2(c) at a time of 50 seconds as low frequency 
energy smearing into the higher frequencies in the spectrogram, with a corresponding increase in amplitude in the time-domain.

Contaminations other than those shown in Figure 5.2 may also have been present. Electrical noise could manifest as high amplitude tonals at the hydrophone operation frequency and its harmonics, and/or as Gaussian noise across a wider frequency band. Impact noise from a fish colliding with a hydrophone or something else tapping the hydrophone array would likely be observed as sharp amplitude peaks in the time domain, and energy would be smeared across the frequency spectrum at this time. Signals from any ships in the vicinity of the array throughout the day would be recorded as discrete high amplitude tonals.

All discrete signals have a difference in direct path length to each hydrophone which is less than or equal to the direct path between the hydrophones, and may be visible in the cross-correlation as spurious precursory arrivals. Preprocessing, which includes choice of bandwidth as well as time and frequency domain normalisation, ameliorates this effect (see Sections 5.4.2-5.4.4).

\subsubsection{Spectra and coherence}

Only signals that are received by both hydrophones will sum coherently to give a peak in the cross-correlation function. Spectra give some information for selection of an appropriate bandwidth since signals of very low amplitude or low SNR will generally have poor coherence, which should be avoided; however, higher amplitude does not guarantee higher coherence.

Coherence, $\gamma$, which gives a measure of the degree of linear dependence between two random variables, $X$ and $Y$, as a function of frequency, $f$, is defined by

$$
\gamma^{2}(f)=\frac{\left|E\left(X^{*}(f) Y(f)\right)\right|^{2}}{E(|X(f)|) E(|Y(f)|)},
$$

where $|X(f)|$ is the absolute magnitude of $X(f),{ }^{*}$ denotes the complex conjugate, and $E(X)$ denotes the expected value of a random variable $X$. 
(a)

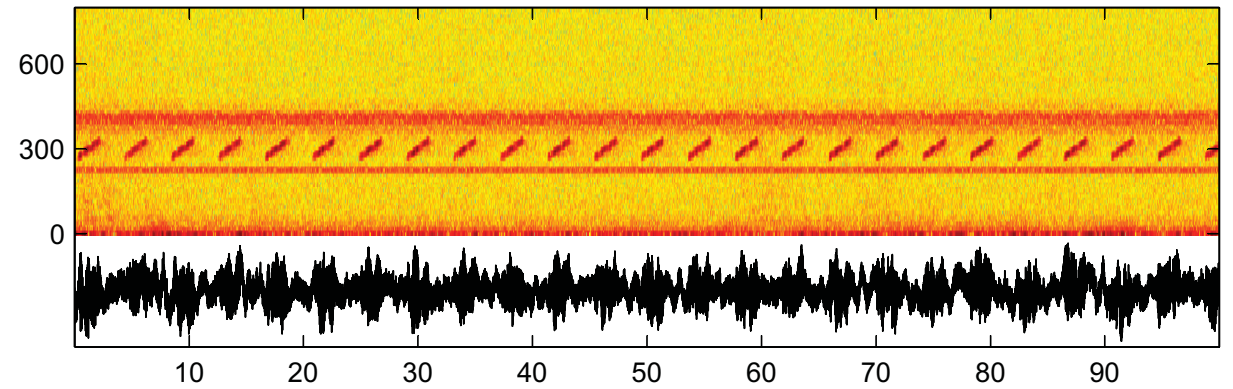

(b)

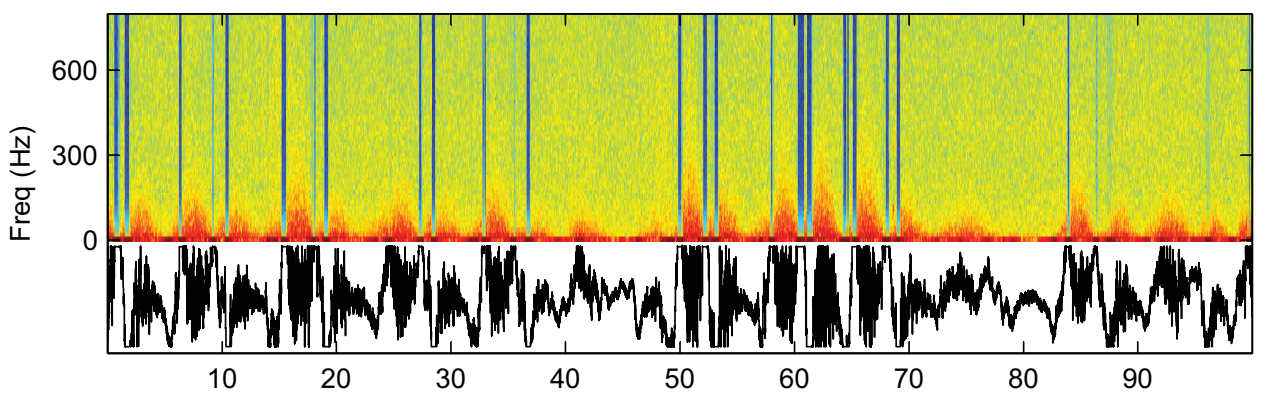

(c)

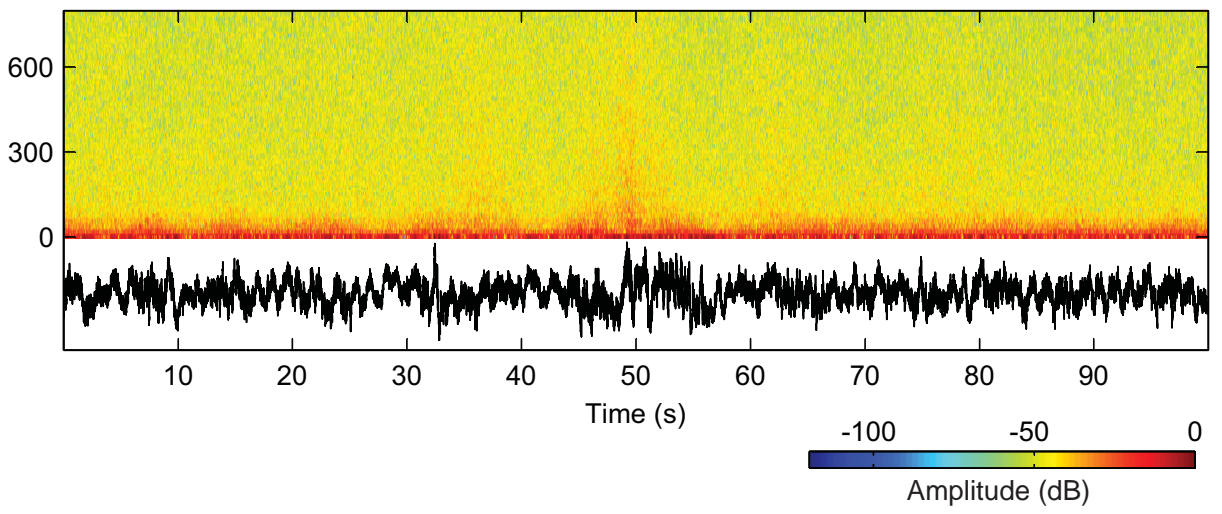

Figure 5.2: Spectrograms (upper) and time-series (lower) of September 2 data: (a) signal dominated by mid-frequency fixed sound sources ( $\mathrm{H}-11$, 0:29:25 Z), (b) clipped signal (H-1, 0:08:56 Z), and (c) energy dominated by low frequency sources (H-11, 12:46:39 Z). Low frequency energy bursts are observable in (b)-(c). Spectrograms (dB) are normalised to the maximum amplitude at each time. 
Both the signal amplitude and coherence are considered here.

Since the underlying statistics of the data are non-stationary, spectra and coherence over short periods are examined. Spectrograms of the signals received by H-52, and coherograms (coherence plotted as a function of frequency and time) of the signals recorded on $\mathrm{H}-52$ and $\mathrm{H}-40$, which are separated by $97.74 \mathrm{~m}$, are shown in Figure 5.3. The mean spectra and coherence for 1:33 min intervals were calculated using 2 second Hanning windowed data segments and $50 \%$ overlap. The mean of the individual spectra and coherences are shown on the far right.

High amplitude signals with a corresponding high coherence are apparent at regular time intervals in the $200-410 \mathrm{~Hz}$ frequency range, as shown in Figure 5.3(a)-(b). These signals are from the three fixed location sound sources described in detail in Section 5.4.1, which were only active for the first ten minutes of every half hour. Low signal amplitudes and coherence are observed at frequencies above $420 \mathrm{~Hz}$, and also from 100-200 Hz. Below $100 \mathrm{~Hz}$ both the amplitude and coherence of the received signals are higher, as shown in Figure 5.3(c). This is expected since lower frequency ocean noise will propagate more coherently over long distances. A banded structure consisting of high amplitude tonals is observed in both the low frequency spectrogram and coherogram, Figure 5.3(c)-(d), at a range of frequencies at different times throughout the day (e.g., 1:30-3:30 Z and 13:30-15:30Z). This banded structure is indicative of the sound field being dominated by ship noise at low frequencies. The exact frequency content of the signals emitted by each ship is dependent upon the propeller frequency as well as other characteristic properties of the ship, and therefore the frequency content of the banded structure changes throughout the day as different ships dominate the overall sound field.

Since the signals, apart from those from the fixed sources, exhibit negligible amplitude and coherence above $100 \mathrm{~Hz}$, the data were bandpass filtered to $20-100 \mathrm{~Hz}$. The lower limit of $20 \mathrm{~Hz}$ was selected as frequencies below this have insufficient resolution over the distances in question. 
Analysis of data preprocessing methods

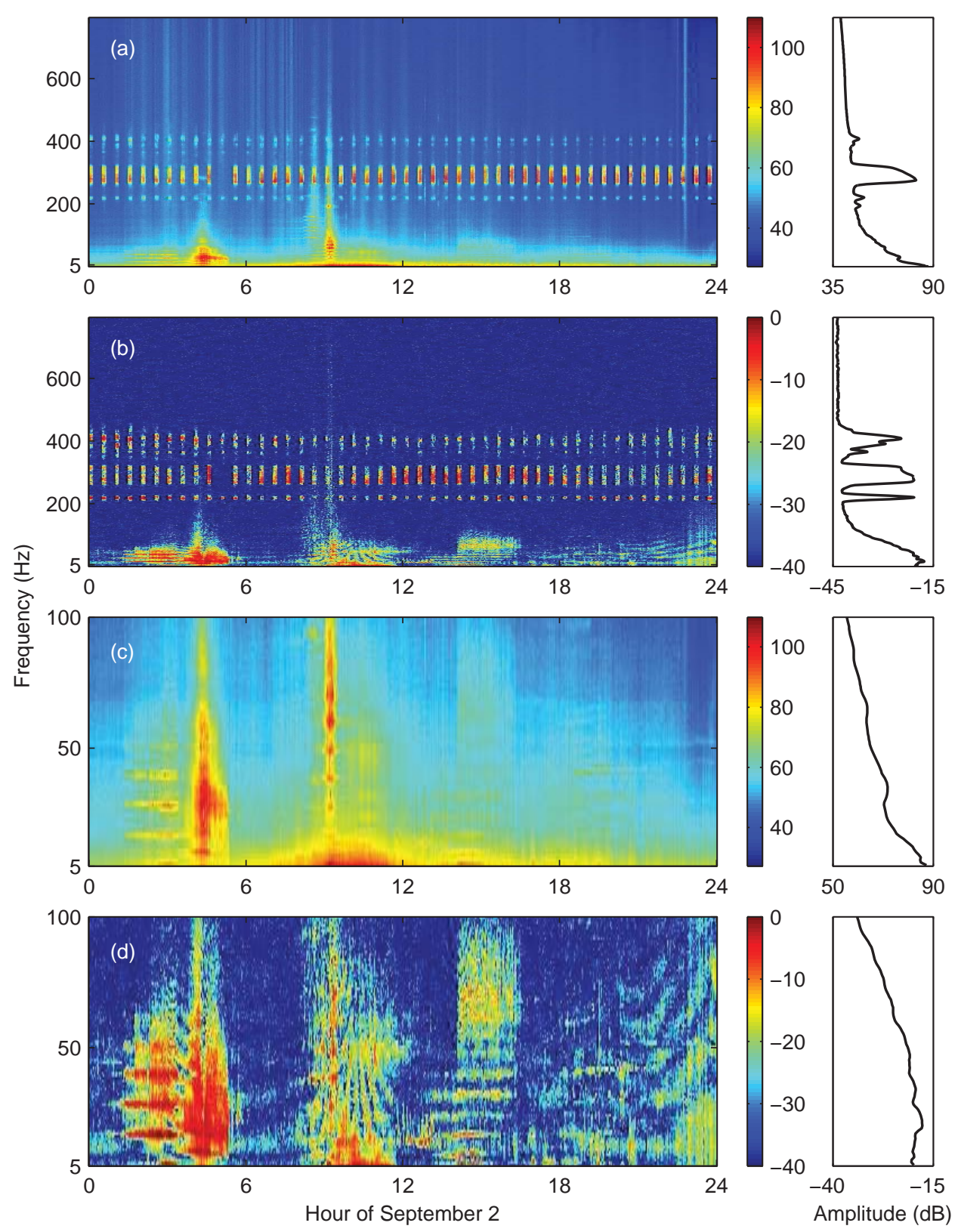

Figure 5.3: Spectrograms (dB) of signals recorded on H-52, using the entire September 2 data: (a) 5-800 Hz and (c) 5-100 Hz. Coherograms (dB relative to unity linear coherence) of the data recorded on $\mathrm{H}-52$ and $\mathrm{H}-40$ : (b) $5-800 \mathrm{~Hz}$ and (d) $5-100 \mathrm{~Hz}$. The average of the individual spectra or coherences is plotted to the right of each figure. 


\subsubsection{Frequency domain normalisation}

Frequency domain normalisation has the dual purpose of broadening the signal bandwidth by placing higher emphasis on low amplitude signals, and of decreasing the negative impact of discrete sources. For the purpose of comparing frequency domain normalisation methods, the effects of erroneous temporal peaks were minimised by setting all values of amplitude greater than $50 \%$ of the signal standard deviation to this value [16, 81], a process described as 'threshold clipping', one of the time domain normalisation techniques that is discussed in detail in Section 5.4.4.

\section{Normalisation methods}

Cross-correlations were performed using five different frequency domain preprocessing methods:

(a) no frequency domain preprocessing;

(b) bandpass filter, no frequency domain amplitude normalisation;

(c) bandpass filter and whiten by normalising over the entire frequency range $(20-100 \mathrm{~Hz})$, known as absolute whitening;

(d) bandpass filter and normalise by a smoothed version of the amplitude spectrum, known as smoothed whitening; and

(e) bandpass filter and partially normalise the data by the sum of the signal magnitude at that frequency and a mean amplitude dependent constant:

$$
S(\omega)=\frac{S(\omega)}{|S(\omega)|+\beta \overline{|S|}}
$$

where $\overline{|S|}$ is the mean amplitude over the entire frequency range, and $\beta$ determines the degree to which the data are whitened $(\beta=0$ is equivalent to absolute whitening (c), and $\beta=\infty$ to no normalisation(b)). 
Analysis of data preprocessing methods

The effect of applying each normalisation technique to $\mathrm{H}-40$ data can be seen in Figure 5.4. Bandpass filtering without frequency normalisation, method (b), maintains the general characteristics of the amplitude peaks and decay with frequency seen in the raw data, method (a). Absolute and smoothed whitening, methods (c) and (d) respectively, give approximately equal energy across the frequency band. Partial whitening, method (e), reduces extraneous tonals, but also places emphasis on signals of higher coherence (lower frequency).

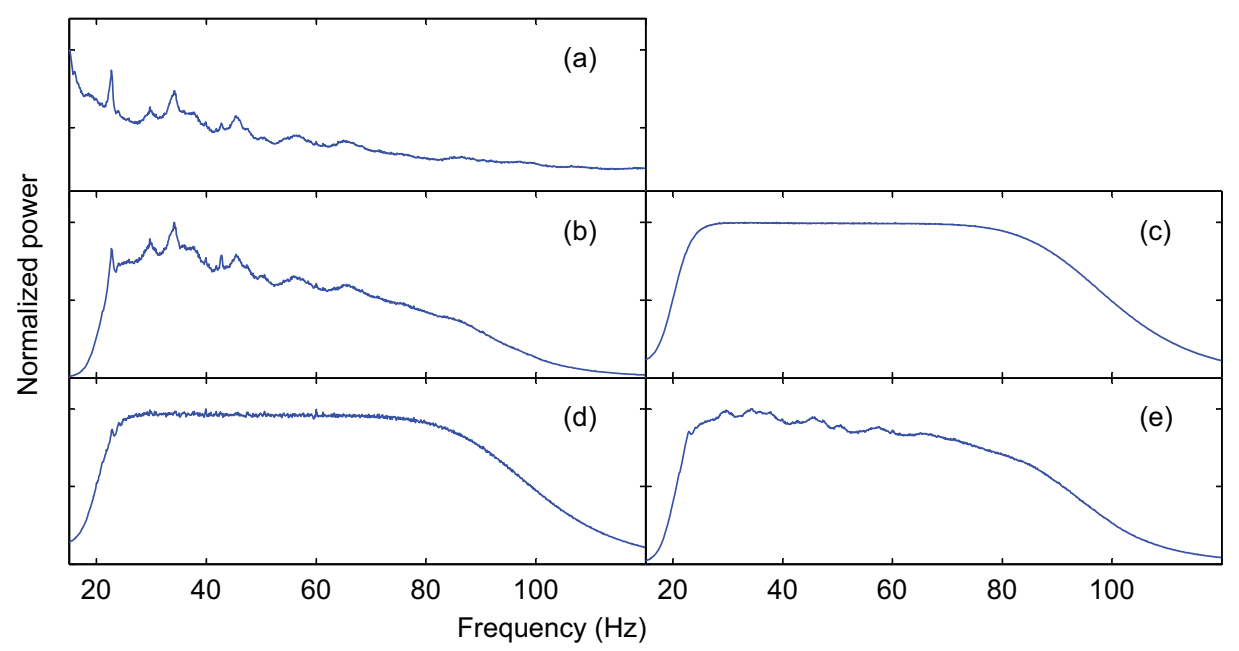

Figure 5.4: Normalised (linear) spectra of the September 2 signal recorded on H-40 (a) before pre-filtering, and (b)-(e) after pre-filtering. Pre-filtering methods are (b) bandpass and time domain filtering only, (c) absolute whitening, (d) smoothed whitening, and (e) partial whitening $(\beta=1)$.

\section{Cross-correlations}

Data were pre-processed using each of the methods outlined in the previous section. Individual cross-correlations were calculated and normalised by their peak value before summing so that the overall cross-correlation is not dominated by high amplitude cross-correlations from only part of the day. The cross-correlations between H-52 (tail-end HLA hydrophone) and all 
other hydrophones, summed over September 2, using smoothed whitening filtering, method (d), are shown in Figure 5.5(a).

The HLA cross-correlations are plotted as a function of distance from $\mathrm{H}-$ 52. The VLA cross-correlations, which are offset by the horizontal distance between H-52 and the VLA hydrophones, are plotted as a function of height from the seafloor (note that the two vertical axes have different scales). The direct and surface reflected travel times between each hydrophone, which are shown as dotted lines, were determined using OASES [49]. Peaks in the cross-correlation are evident at both the direct and surface reflected travel times. The EGFA envelope in Figure 5.5(b), on a logarithmic scale, reveals the surface-bottom reflected path to the lower VLA hydrophones.

The EGFA envelope for the case of bandpass filtering only, method (b), shown in Figure 5.5(c), shows only minor differences to that for smoothed whitening. Due to the higher proportion of low frequency energy, the arrivals are less sharp, and the background noise level is slightly higher. In addition, the surface reflected path is not as clear at the closer hydrophones (40-120 m). The raw signals have greater amplitude at lower frequency and this naturally assists the cross-correlation when no frequency domain normalisation is applied; the lower coherence signals are lower amplitude and will therefore have less overall influence upon the correlated signal. This explains why a reasonable cross-correlation can be determined when no spectral normalisation is performed. The EGFA envelope for no frequency domain filtering, method (a), shown in Figure 5.5(d), gives a poor representation of the Green's function. A low frequency signal below $20 \mathrm{~Hz}$ from the southeast dominates the cross-correlation to such an extent that only the direct acausal path is obtained.

The EGFA envelopes for absolute and partial whitening, methods (c) and (e), are not shown, but their characteristics lie between that of Figure 5.5(b) and (c). Smoothed whitening was selected as the optimal frequency domain filtering method for the data collected. Bensen et al. [62] compared no normalisation and smoothed whitening for cross-correlations 

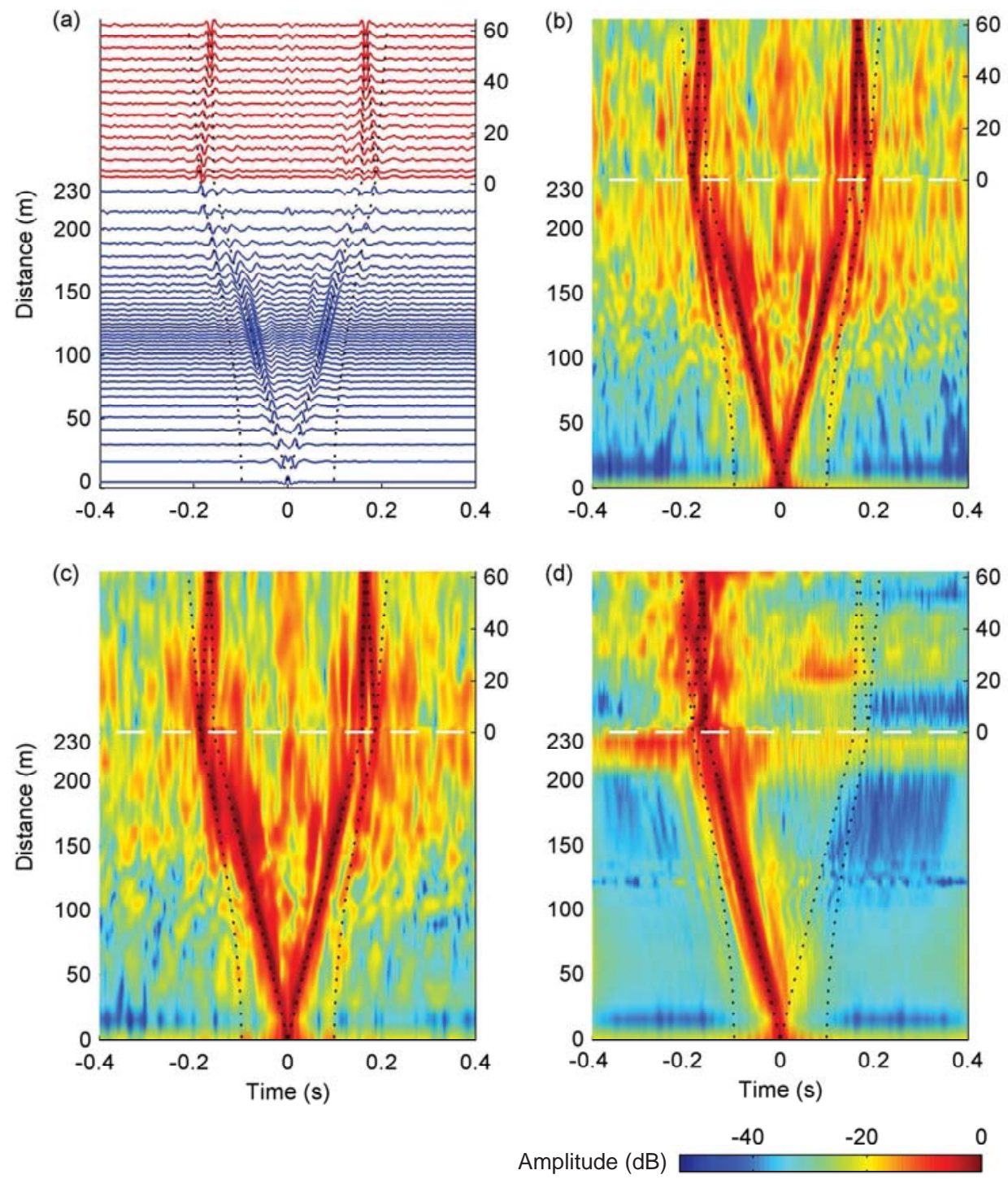

Figure 5.5: (a) Cross-correlations between H-52 and all other hydrophones for September 2 data using smoothed-whitening frequency filtering (20$100 \mathrm{~Hz}$ bandwidth). (b)-(d) EGFA envelopes (dB relative to maximum value): (b) with smoothed whitening (20-100 Hz bandwidth), (c) with no frequency normalisation (20-100 Hz bandwidth), and (d) with no frequency domain filtering or normalisation. The lower traces are cross-correlations with HLA hydrophones; their distances from the tail hydrophone (H-52) are shown on the left side axis. The upper traces are cross-correlations with VLA hydrophones; their vertical distances from the seafloor are shown on the right side axis, which is offset by the horizontal distance of the VLA from the HLA tail. The simulated travel times between the hydrophones were calculated using OASES and are overlaid as dotted lines. 
of seismic data, which were created by different physical processes and have much lower frequencies. They found that the improvements gained by normalisation were substantially greater than for the data considered here.

The envelopes of the EGFAs generated using the various frequency domain normalisation techniques were compared here; however, it should be noted that because these normalisation methods are non-linear, not only the amplitude, but also the phase of the EGFAs are affected. The variations in phase due to the application of each technique were found to be small, and the only conclusive evidence that could be ascertained was that the greater the degree of non-linearity in the normalisation, the greater the effect on the phase of the obtained arrivals. The method of smoothed whitening used here has only a relatively small degree of non-linearity, and therefore should not significantly affect the arrival time.

Appropriate selection of the data bandwidth affected the result more than any frequency domain normalisation because above the $100 \mathrm{~Hz}$ lowpass frequency the cross-correlation has almost no coherence, and therefore inclusion of higher frequencies adds to the noise floor. If no frequency domain filtering or normalisation is applied this added noise is minimal, since the amplitude is negligible at higher frequencies. However, if the data are whitened but not bandpass filtered, signals of low-coherence will be emphasised, and the resulting cross-correlation sum will be dominated by noise that requires very long averaging times to remove.

\subsubsection{Time domain normalisation}

\section{Theory}

Various methods of time-domain normalisation have been used by others. Campillo and Paul [12] cross-correlated seismic coda. Since the coda decays over time, the overall cross-correlation would be naturally biased toward the earlier part of the coda. To compensate for this they used short segments of data, performed the cross-correlations, normalised these, and then summed 
Analysis of data preprocessing methods

them. They reported results similar to those obtained by others [82] who had used one-bit normalisation, where only the sign (phase) of the waveform, not the amplitude, is retained. One-bit time reversal normalisation yields a higher SNR than classical time reversal in some multiple scattering or reverberating media $[59,82]$ since all scattering paths are equally weighted. Without one-bit normalisation longer scattering paths would have a reduced amplitude in the cross-correlation. A similar argument holds for cross-correlation analysis and therefore one-bit normalisation is often used $[27,59,83]$. Another method of time domain normalisation is to clip all signals above a certain threshold [81]. This minimises the effect of energy bursts, but also maintains more information than 1-bit normalisation. Gerstoft et al. [16] set their threshold as the minimum of the standard deviations measured over each day. For their data set this gave identical results to one-bit normalisation. Bensen et al. [62] and Yang et al. [18] used temporally variable weighting functions. They claimed that these retain more small amplitude information and also allow for flexibility in defining the amplitude normalisation in particular frequency bands.

Comparisons of time domain normalisation techniques by Bensen et $a l$. [62] concerned seismic noise, which is often dominated by high amplitude earthquakes. Since ocean noise is dominated by other physical processes, which display different characteristics, the effect of these normalisation techniques upon the resulting cross-correlations will also differ. A comparative study of the applicability of several techniques to the current data set was therefore undertaken. The normalisation techniques that were compared are:

- no normalisation;

- correlate over short intervals with some degree of overlap, normalise the cross-correlations and then sum;

- clip the signal to a threshold;

- one-bit (two level) normalisation; 
- use of a rectangular central temporally variable weighting (RCTVW) function; and

- use of an exponential central temporally variable weighting (ECTVW) function.

Performing no normalisation in the time domain sets a clear benchmark for the five other techniques. Cross-correlating over short intervals and then summing the normalised cross-correlations is more effective for shorter intervals. Since the greatest distance between any two hydrophones is $230 \mathrm{~m}$, the direct path should be observable in under $0.2 \mathrm{~s}$; hence, to ensure sufficient time for reverberant paths to be captured, $0.4 \mathrm{~s}$ data segments were used, with $33 \%$ overlap.

A threshold of $\sigma$, one standard deviation, was chosen as the level to which the signal would be clipped for normalisation technique (c). It was noted that the results were not highly sensitive to the chosen threshold.

One-bit normalisation, which uses only the sign of the signal, increases the signal-to-noise ratio of the data:

$$
s_{n}(t)=\left\{\begin{array}{cl}
-1 & \text { if } s(t)<0 \\
1 & \text { if } s(t)>0
\end{array}\right.
$$

where $s(t)$ is the raw signal at time $t$, and subscript $n$ denotes the normalised signal.

RCTVW and ECTVW are the most computationally time intensive. RCTVW normalises each point by the sum of the unweighted mean of the absolute value of $N$ preceding and succeeding values $(2 N+1$ points overall):

$$
s_{n}(t)=\frac{s(t)}{w(t)}
$$

where

$$
\begin{aligned}
w(t) & =\sum_{\tau=t-N}^{t+N}|s(\tau)| \\
& =w(t-1)-|s(t-N-1)|+|s(t+N)| .
\end{aligned}
$$


Analysis of data preprocessing methods

A normalisation window of $0.05 \mathrm{~s}$, which is the time interval of the maximum period, corresponding to the minimum frequency of $20 \mathrm{~Hz}$, was found to be suitable. A normalisation vector length of $2 N+1=257$ was therefore used.

ECTVW places more emphasis on points closer to the point of interest. It normalises in the same manner as RCTVW, the only difference being that it applies a weighting filter with an amplitude that decreases exponentially in both directions from the data point of interest:

$$
\begin{aligned}
w(t)= & (1-\alpha)^{N}|s(t-N)|+\ldots \\
& +(1-\alpha)|s(t-1)|+|s(t)|+(1-\alpha)|s(t+1)|+\ldots \\
& +(1-\alpha)^{N}|s(t+N)|
\end{aligned}
$$

where $\alpha=2 /(N+1)$ is the exponential smoothing factor. In order to use previously calculated sums to determine subsequent weights, the exponential is split up into two parts, the increasing exponential prior to and including the point, and the decreasing exponential after the point. These are then summed to give the overall weighting.

\section{Application to data}

Example waveforms resulting from application of each time normalisation method to 2.5 s of H-40 data are shown in Figure 5.6. Higher energies are observed in the time period 1.4-2.1 s, as can be seen in Figure 5.6(a), and these amplitudes are all successfully reduced by the time-filtering methods, as shown in Figure 5.6(b)-(e). Normalisation technique (b) is not shown here as this normalisation is only applied after cross-correlating the data.

Cross-correlations and EGFA envelopes for September 2 for each timenormalisation method are shown in Figure 5.7(a)-(b). The same line style has been used in the figure for all results because they are too similar to be individually discerned. The horizontal distance between: (a) the HLA hydrophones H-52 and H-48, is $31.31 \mathrm{~m}$; and (b) H-52 and H-8 (located in the VLA), is $230 \mathrm{~m}$. Large cross-correlation peaks exist at the direct ray travel time, and smaller peaks at the surface reflected travel time. The 


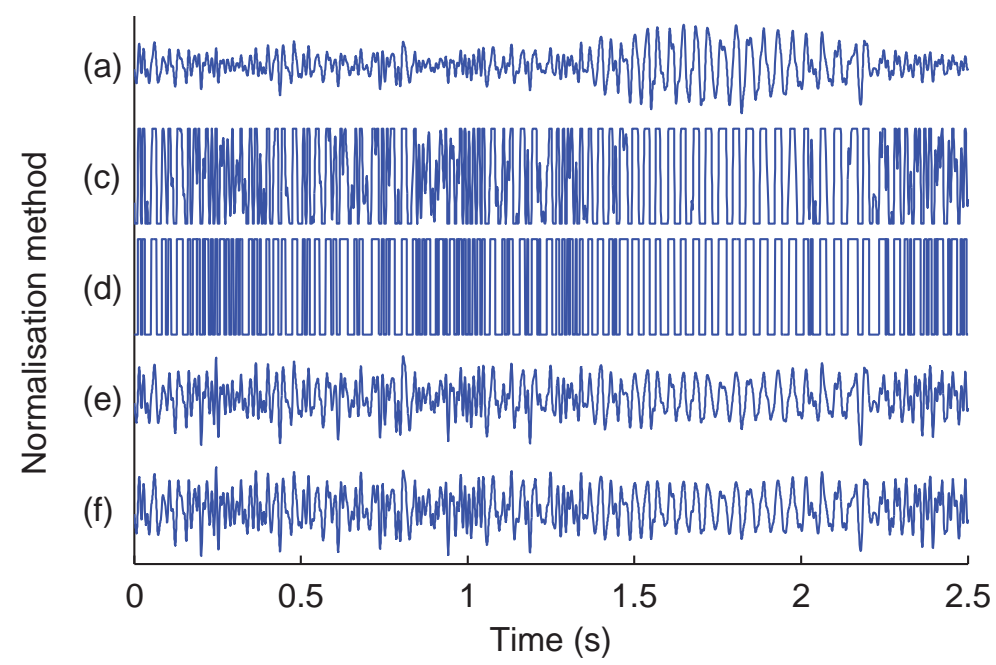

Figure 5.6: Preprocessed waveforms for $2.5 \mathrm{~s}$ of $20-100 \mathrm{~Hz}$ bandpass filtered data from H-40 (at 12:48:45 Z) with normalisation method: (a) none, (c) threshold clipping, (d) one-bit, (e) RCTVW, and (f) ECTVW.

background noise is consistently low, except for one high peak at a time just less than the positive direct arrival. This could be due to a nonuniform source distribution. For the two further spaced hydrophones shown in Figure 5.7(b), the EGFA envelope once again peaks at the direct and surface reflection travel times. A smaller peak can also be seen at the acausal surface-bottom travel time (i.e., the bottom-surface reflected path from H-8 to H-52). The signal-to-noise ratio is poorer than for the more closely spaced hydrophones, but this is to be expected since decay and spreading of signals increases with distance.

The results from Figure 5.7(a)-(b) suggest that time-normalisation has little influence on the cross-correlations for this data set. Time normalisation is important for seismic cross-correlations [61, 62] since otherwise the results can be dominated by earthquakes. Although the ocean noise field is not perfectly diffuse, there are no equivalently energetic events for the frequency band considered, and nearby shipping is minimal on September 2. This, combined with the intrinsic averaging introduced by summing over the entire day, are two reasons why time-domain normalisation shows 

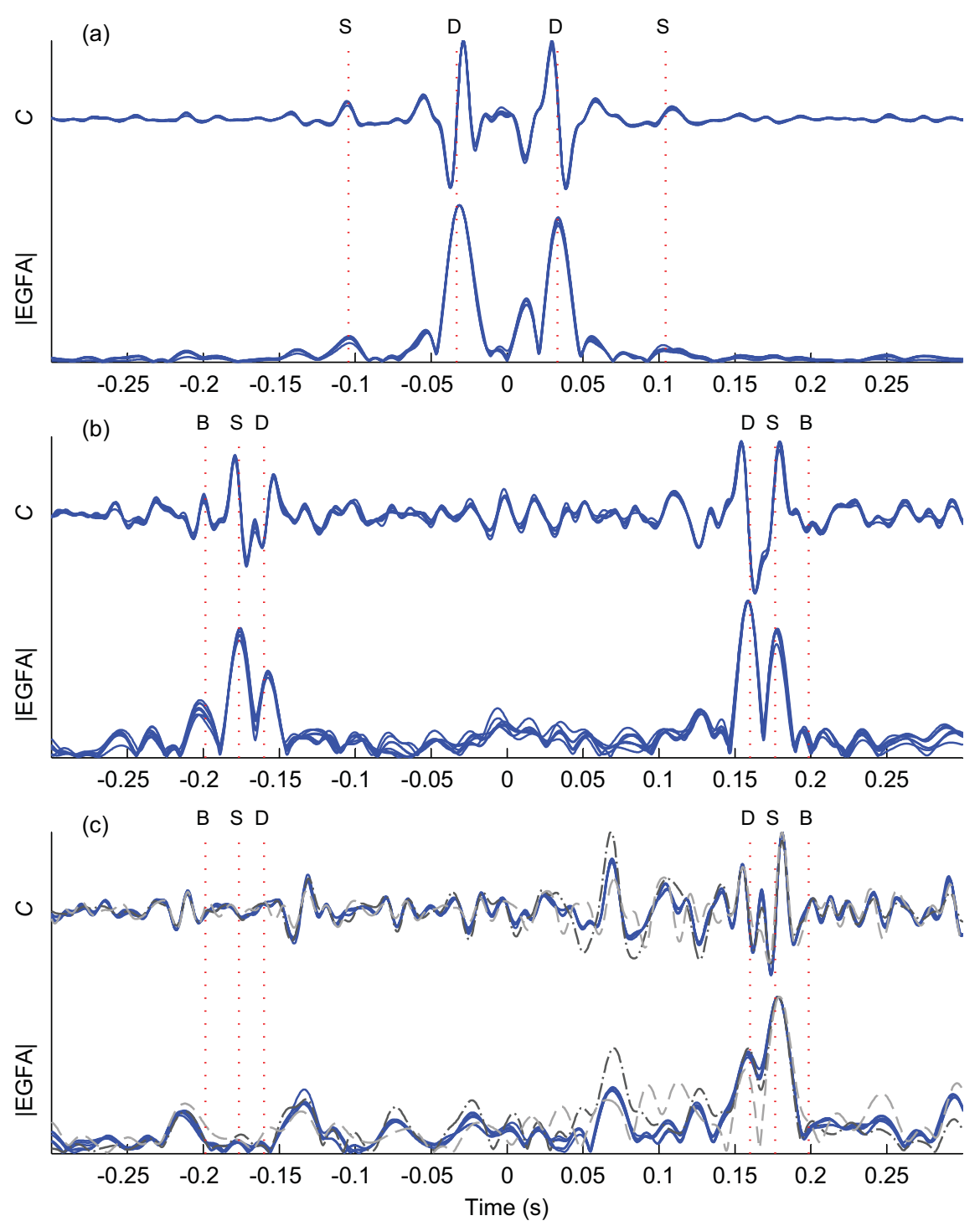

Figure 5.7: Summed cross-correlations, $C$, and EGFA envelopes, |EGFA|, for all time normalisation methods for H-52 and (a) H-48 (entire day with $51.32 \mathrm{~m}$ horizontal separation), (b) H-8 (entire day with $230 \mathrm{~m}$ horizontal separation), and (c) H-8 (10:24 min from 8:30 Z). Simulated travel times of direct (D), surface (S), and surface-bottom (B) paths are shown as vertical dotted lines. In (c) results for no normalisation and short interval cross-correlations are shown as dark grey dash-dotted and light grey dashed respectively. 
negligible benefit here.

If the cross-correlations were carried out over a period that is insufficient to average-out energetic events, the benefits of normalisation would be greater. Consider the 10:14 min cross-correlation between $\mathrm{H}-52$ and $\mathrm{H}-$ 8 in Figure 5.7(c). The cross-correlations peak at the positive direct and surface reflected travel times only, indicating that the dominant sound field is from the tail-end of the array (the north-west direction). Distinct peaks seen at $-0.22,-0.14$, and 0.07 seconds are the result of discrete sources. Since high amplitude events, which are reduced in the normalisation process, are not averaged-out in the shorter cross-correlation time period, the cross-correlation and EGFA envelope without normalisation, method (a), and correlating over short periods and summing the normalised results, method (b), both have a higher noise level than the results for data that is normalised before cross-correlation.

Since time-domain normalisation techniques (c)-(f) all give similar results, and one-bit normalisation is the least computationally intensive, it was selected for further processing and analysis of the data.

The sum of the cross-correlations between H-52 and all other hydrophones for $20-100 \mathrm{~Hz}$ bandpassed, one-bit normalised, smoothed whitened September 2 data are shown in Figure 5.8(a). Since the Green's function is related to the time-derivative of this (the EGFA), the flipped in time acausal EGFA, which is clearer than the causal signal, is plotted in Figure 5.8(b). The Green's function, which was simulated using OASES and then convolved with a $20-100 \mathrm{~Hz}$ box car pulse, is shown in Figure 5.8(c) for comparison purposes. The assumed model sediment properties, $c=1761 \mathrm{~m} / \mathrm{s}$ and $\rho=1.69 \mathrm{gm} / \mathrm{cm}^{3}$, were approximated from grab samples in the array vicinity [73]. In both cases the direct arrival peaks are positive and the reflected arrival peaks are negative, which is due to the phase change at the surface. The amplitudes are not exact, though this is expected since the acoustic energy is not equi-partitioned amongst all modes [84].

The EGFA envelope shown in Figure 5.8(d) shows the arrivals more 
clearly. The surface-reflected arrivals are apparent for distances greater than about $50 \mathrm{~m}$, as indicated by the peak in EGFA amplitude that occurs above $50 \mathrm{~m}$ in the figure at times corresponding to the second set of dotted arrivals on either side of zero time. They are not observable at closer ranges, where they would be more steep, because at these ranges the noise field is dominated by far-field horizontally travelling wavefronts.

If a cross-correlation is started or finished part way through a ship's track, the cross-correlation may be biased. Tapering of the cross-correlation amplitudes towards the start and end of the cross-correlation was therefore considered; however, for the given data set and long cross-correlation times, tapering was seen to have little effect.

\subsection{Geometric comparisons}

Examples of EGFA envelopes with respect to hydrophones other than the outer-most HLA hydrophone, H-52, are shown in Figure 5.9. Due to the steeper grazing angle (of the acoustic paths relative to the horizontal) to the furthest hydrophone, cross-correlations with H-34, a central HLA hydrophone, shown in Figure 5.9(a), do not yield as much information about the surface reflected path as do cross-correlations with H-52, shown in Figure 5.8(d). Figure 5.9(b) reveals that cross-correlations with VLA H-10 show the surface reflected path, at slightly larger times than the dominant direct path, for distances of $0-150 \mathrm{~m}$ from the tail end of the HLA; however, the surface path is not as clear as that obtained when correlating with H-52, which is likely due to either the decreased stability in the environment at the shallower depth of $\mathrm{H}-10$, or the increased motion of the VLA hydrophones relative to the HLA hydrophones. The bottom-surface reflected arrival from $\mathrm{H}-10$ to the HLA hydrophones is also observable at a time just after the surface reflected path, but the acausal path from the HLA to H-10 is not observable.

The September 2 EGFA envelopes for SWAMI32 and Shark are shown 


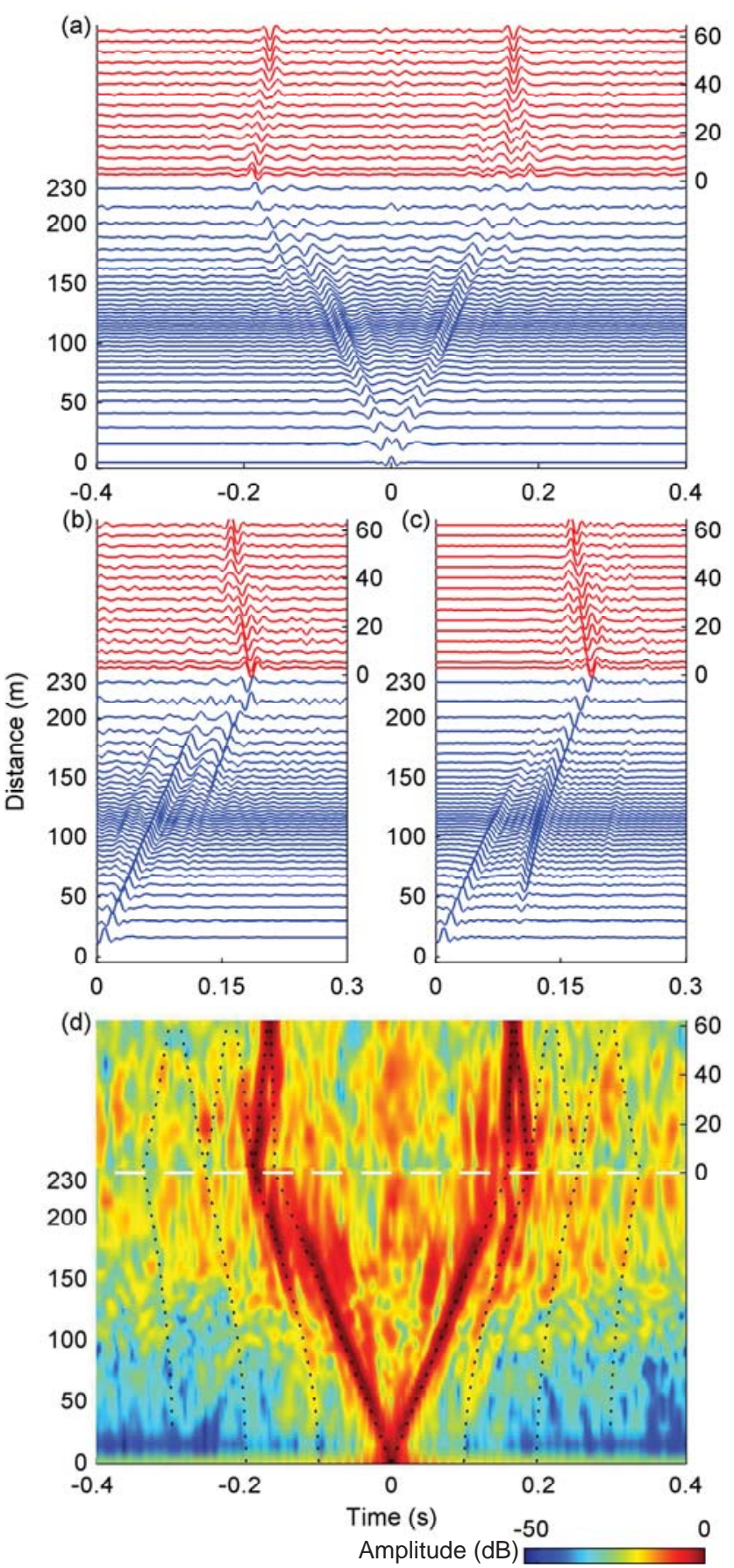

Figure 5.8: September 2 (a) summed cross-correlations and (b) EGFAs between H-52 and all other hydrophones. (c) Simulated Green's functions convolved with a $20-100 \mathrm{~Hz}$ bandwidth linear source. (d) EGFA envelopes (relative to maximum value) with simulated inter-hydrophone travel times overlaid as dashed lines. Vertical axes format is the same as in Figure 5.5. 

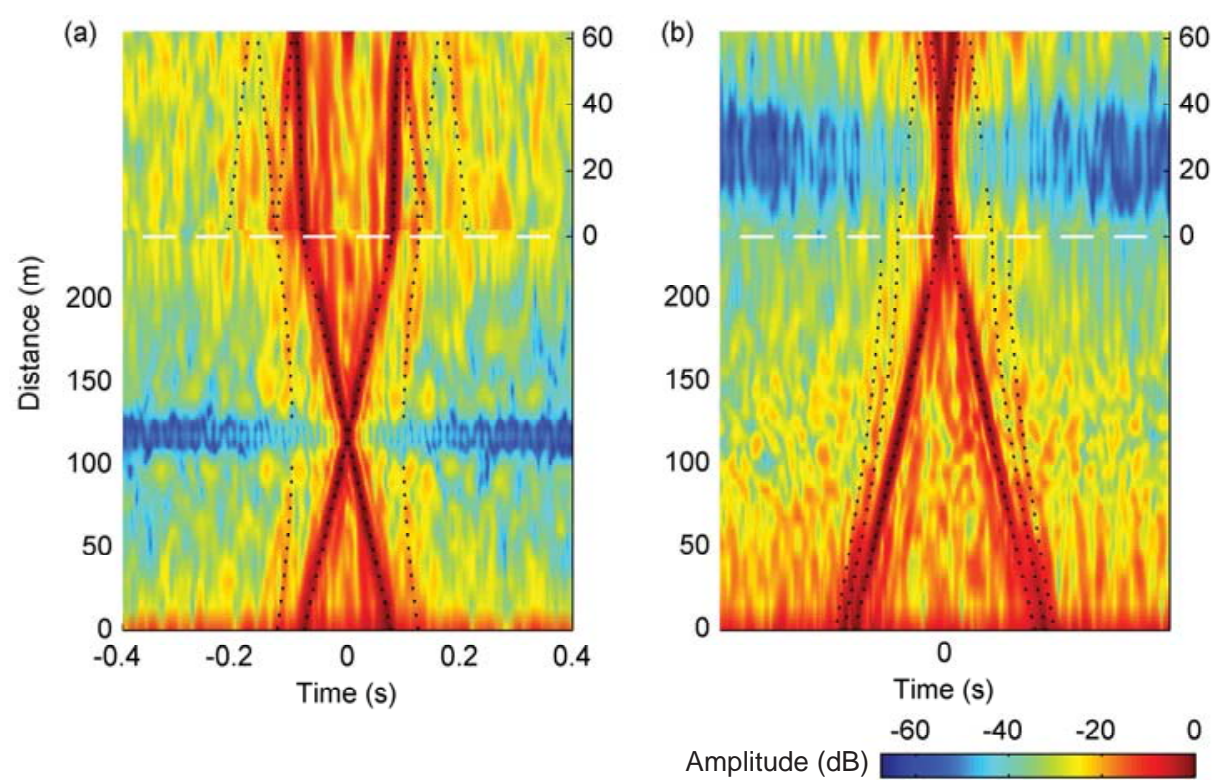

Figure 5.9: September 2 EGFA envelope ( $\mathrm{dB}$ relative to maximum value) with respect to: (a) H-34, and (a) H-10. Vertical axes are the same as in Figure 5.5.

in Figure 5.10. The SWAMI32 cross-correlations are with respect to H-30 rather than the tail hydrophone due to high noise on the outer two hydrophones. The high noise levels are attributed to channel switching, which is discussed extensively in Chapter 7. Like the SWAMI52 results shown in Figure 5.8(d), the SWAMI32 and Shark array cross-correlations show both the direct and surface reflected paths. All the SWAMI and Shark results in Figures 5.8-5.10 show that although the direct path dominates for more closely spaced hydrophones, the relative amplitude of the surface reflected path increases at greater distances. These relative amplitudes depend upon array geometry, modal distribution of acoustic energy, roughness at the surface and, importantly, the impedance at the seafloor. As such, a relationship between the relative amplitudes of the paths and the critical angle could potentially be determined.

Unlike the tapered spacing of the SWAMI array HLA hydrophones, the Shark HLA hydrophones are evenly spaced at $15 \mathrm{~m}$ intervals. Cross- 

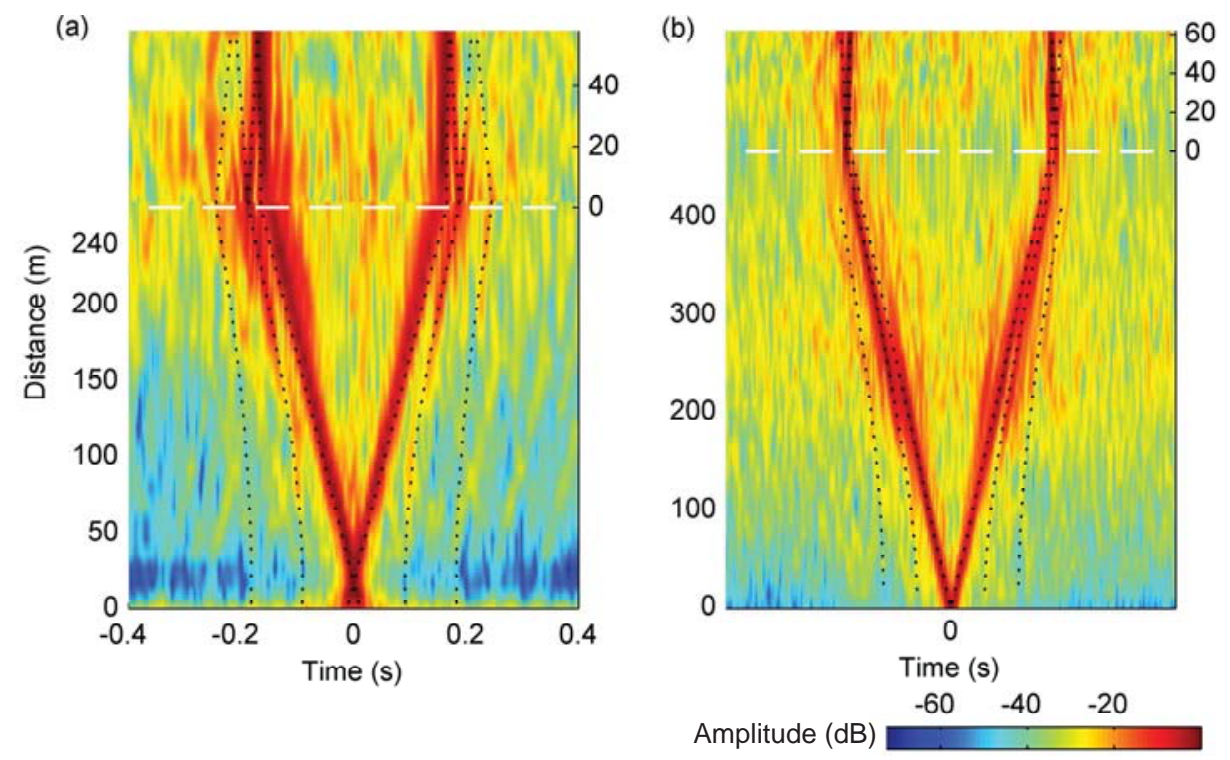

Figure 5.10: September 2 EGFA envelopes (dB relative to maximum amplitude) for (a) SWAMI32, with respect to H-30, and (b) Shark, with respect to H-16. Vertical axes format is the same as in Figure 5.5.

correlations between hydrophone pairs with the same separation distance and direction were compared. The September 2 cross-correlation sums between all HLA pairs separated by $345 \mathrm{~m}$ are plotted in Figure 5.11(a). The traces are similar, and all display cross-correlation peaks at approximately $\pm 0.24 \mathrm{~s}$. The median value of the signals is plotted against a shaded area encompassing the range of all signal values in Figure 5.11(b). A magnified view of part of the signal is provided in Figure 5.11(c) and shows that the signal variation is minimum near the direct path travel time. Summing the cross-correlations of all equally spaced hydrophone pairs should therefore increase the signal-to-noise ratio of the data. The EGFA envelopes between H-52 and all other HLA hydrophones are compared to EGFA envelopes calculated from the sum of the cross-correlations for all pairs at each spacing in Figure 5.11(c)-(d). The overall signal-to-noise ratio is seen to increase when the median cross-correlation is used. Note that the relative amplitudes at some path times not corresponding to inter-hydrophone travel times of the ocean waveguide paths (e.g., $t=0.08 \mathrm{~s}, D=200 \mathrm{~m}$ ) do not decrease. These 
signals could be from sediment paths or reflections from deeper layers, or they could be non-Green's function arrivals that are due to the noise being azimuthally inhomogeneous.

(a)

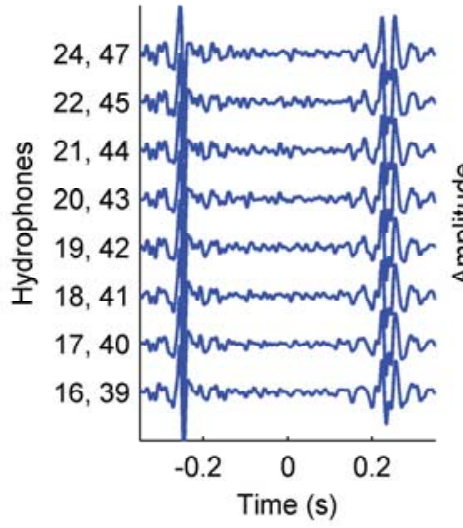

(d)

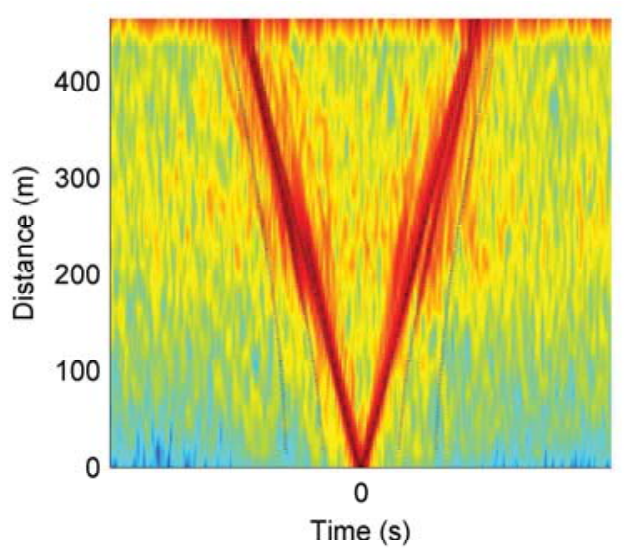

(b)

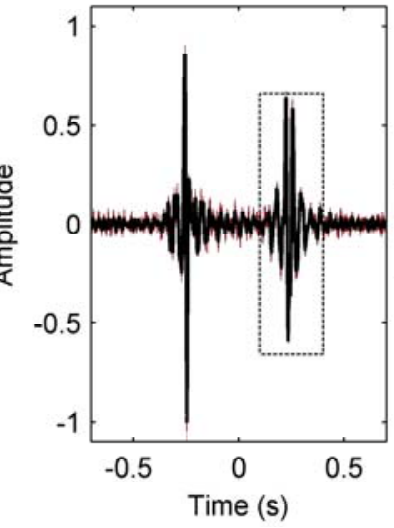

(e)

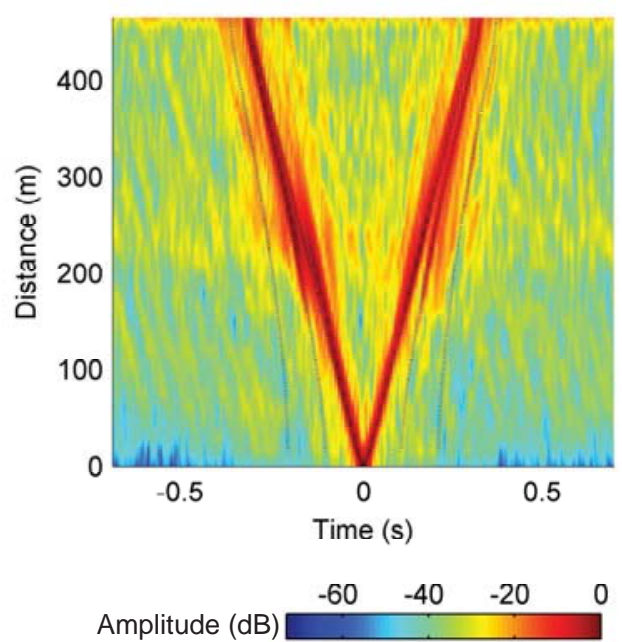

Figure 5.11: (a) Summed cross-correlations over September 2 for Shark hydrophone pairs separated by $345 \mathrm{~m}$. (b) The median cross-correlation from (a) overlies a shaded region between which all values lie. (c) Data from the dashed box in (b) are magnified. EGFA envelopes (dB relative to maximum amplitude) are shown in (d) using cross-correlations between H-52 and all other HLA hydrophones only, and in (e) using the median cross-correlations for all HLA hydrophone pairs. 


\subsection{Temporal variations}

The September 2 data cross-correlations with H-52 were compared with those from adjacent days, and are shown in Figure 5.12. The September 2 data, shown in Figure 5.12(c), peak at the expected direct and surface reflected paths, and exhibit the least background noise. Results from September 1, shown in Figure 5.12(b), are not as clear but are still better than from September 3, shown in Figure 5.12(d), and August 31, shown in Figure 5.12(a), suggesting that the sound field is more diffuse during the storm. Noise from nearby shipping is reduced since most ships retreated from the area for the storm duration, but the overall signal amplitudes are actually higher due to the increase in sea states.

Short time cross-correlations were calculated for data for all three arrays from 0 Z August 31 through 12 Z September 3. SWAMI52 hydrophones H-52 and H-17, SWAMI32 hydrophones H-30 and H-15, and Shark hydrophones H-16 and H-35 were chosen as their separation distances are all similar (between 200 and $285 \mathrm{~m}$ ). Time segments corresponding to one data file were used for SWAMI52 and SWAMI32 (10:14 and 6:24 min respectively), and quarter file (8:34 min) data segments were used for Shark. These time segments differ as it is easier to use only one rather than parts of two files for a single calculation. The times are all sufficiently short for the study of temporal characteristics. The corresponding EGFA envelopes are plotted as a function of time in Figure 5.13(a)-(c), along with the EGFA envelope of the summed normalised cross-correlations over the 84 hour period.

The EGFA envelope is dominated by discrete sources, as indicated in Figure 5.13(a)-(c) by the presence of the high amplitude peaks that occur throughout the day at times less than the direct inter-hydrophone acoustic travel times. Hydrophone spectrograms from times corresponding to the largest peaks are dominated by a banded structure indicative of ship noise. As an example, spectrograms of 60 seconds duration from 3:36:40 Z August 31 for SWAMI52 H-52 and SWAMI32 H-30 are shown in Figure 5.13(d)-(e). Noise from a large ship, with a primary tonal at just 

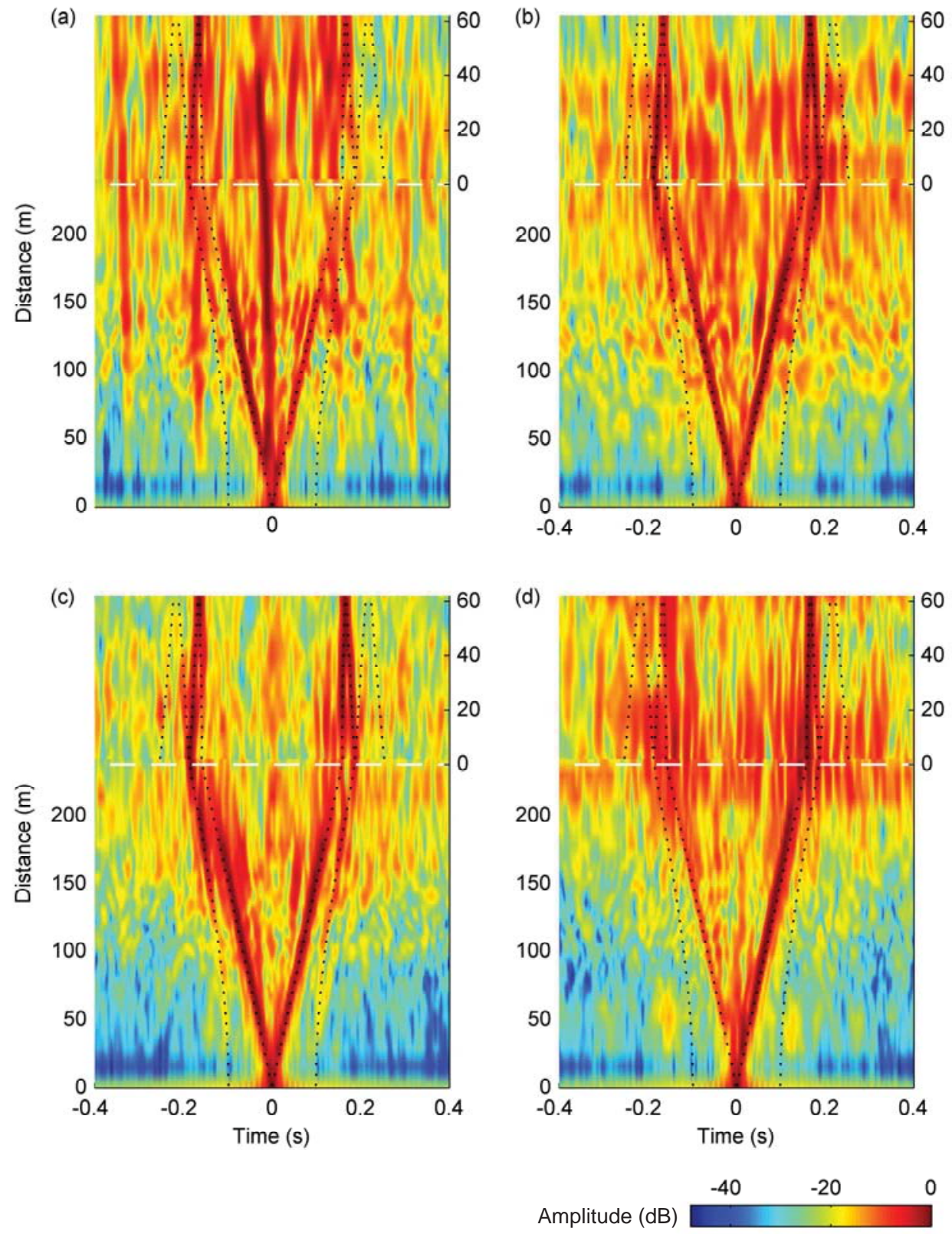

Figure 5.12: EGFA envelope (dB relative to maximum value) with respect to: H-52 for (a) August 31, (b) September 1, (c) September 2, and (d) the first 12 hours of September 3. Vertical axes are the same as in Figure 5.5. 

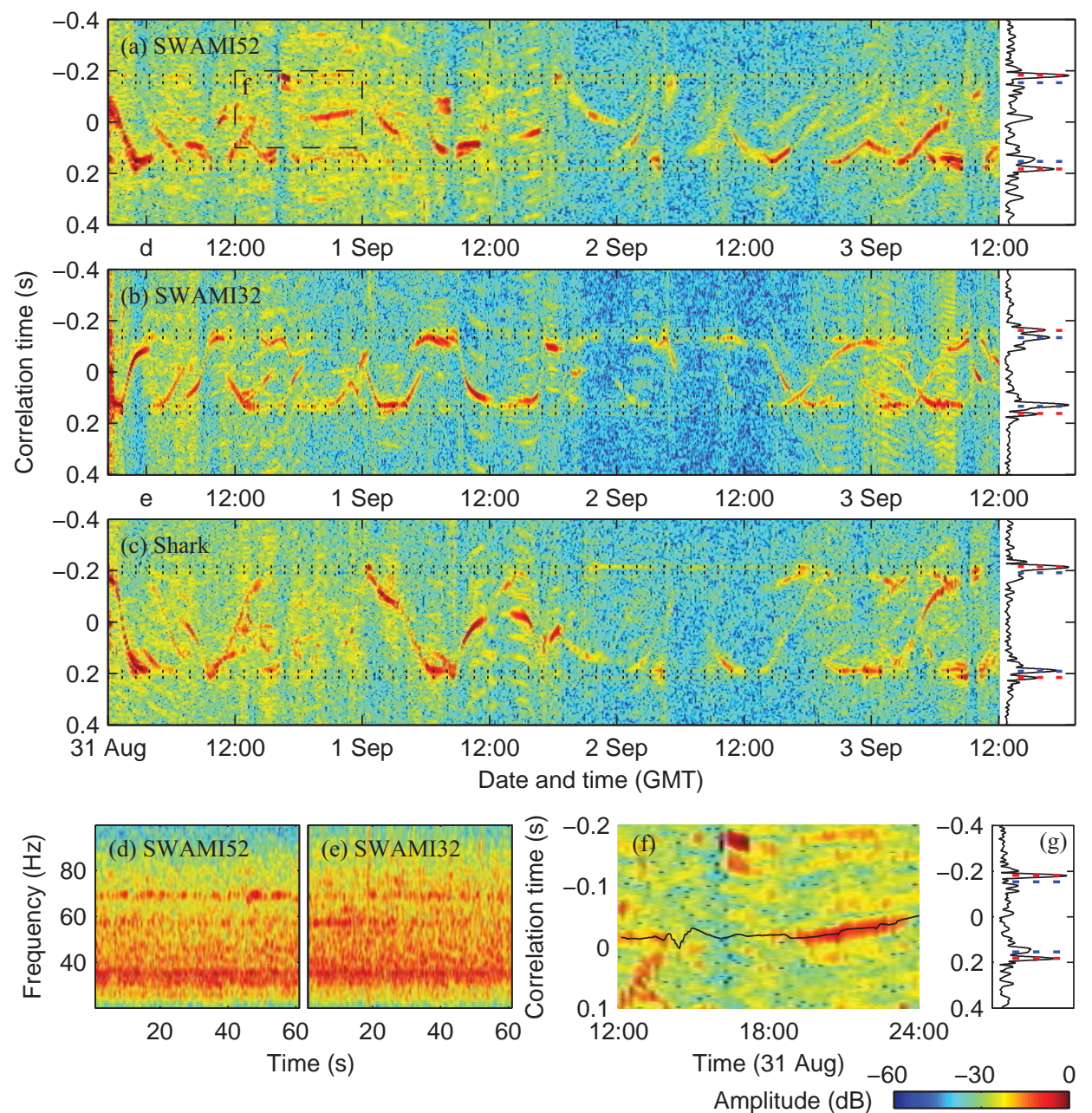

Figure 5.13: EGFA envelope ( $\mathrm{dB}$ relative to maximum value) for: (a) SWAMI52 H-52 and H-17 (230 m separation), (b) SWAMI32 H-30 and H-15 (200 m separation), and (c) Shark H-16 and H-35 (285 m separation). Simulated direct and surface reflected travel times are faintly overlaid as dashed lines. The envelope of the time gradient of the sum of all cross-correlations, normalised by their peak amplitudes to minimise bias from dominant signals, is shown at the right of each plot. (d)-(e) 20-100 Hz spectrograms (dB relative to maximum value) from 3:36:40 Z August 31 for SWAMI52 H-52 and SWAMI32 H-30 respectively (times denoted on (a) and (b) time axes as 'd' and 'e'). (f) Enlarged view of SWAMI52 EGFA envelope, the boxed area from (a), showing a dominant near-side signal, with calculated travel time difference (black line) from R/V Oceanus to the hydrophone pair. (g) The envelope of the time gradient of the sum of all cross-correlations, excluding the period 12-24Z August 31, for SWAMI52 data. A lower threshold of $-60 \mathrm{~dB}$ has been applied to all logarithmic scales. 
Temporal variations

under $40 \mathrm{~Hz}$, dominates both spectrograms. The ship is visible as a peak in the EGFA envelope for all three arrays from 0-4Z August 31. It was ascertained from the time of the EGFA envelope peak that during this period the ship moved from south-west of the arrays, to north of the arrays. The peak in cross-correlation time due to an individual ship changes as a function of the ship's position and hence the signals from a single ship are apparent as curves of high amplitude when plotted as a function of experimental and cross-correlation times. The 'pattern' of curves that is visible in Figure 5.13(a)-(c) is therefore due to a multitude of ship tracks and is entirely dependent on the types and locations of ships passing through the experimental region. Aliasing of the high amplitude ship signals is apparent (e.g., around 12 Z September 1) in Figure 5.13(a)-(c) as lower amplitude replicas of the main EGFA peak at regularly spaced time intervals surrounding the main EGFA peak.

Towards the end of September 1 and on September 2 the EGFA envelope is more stable, as observed by the main peaks in the EGFA being more consistently closer to the dashed inter-hydrophone travel times, and also by the amplitude and number of smaller peaks in the EGFA being reduced. Fewer shipping tracks are seen, and faint arrivals are observable at the inter-hydrophone travel times. This is during the period of high wind, as shown in Figure 5.1(d), and elevated sea conditions from Tropical Storm Ernesto. The reduction in nearby ships and the increase in wave energy results in a greater proportion of acoustic energy in the ocean at these lower frequencies being from breaking waves and cumulative noise from distant shipping, and therefore the noise field is more diffuse. During this period faint peaks are frequently observed at times corresponding to the simulated surface reflection travel times, such as between $22 \mathrm{Z}$ September 1 and $3 \mathrm{Z}$ September 2 in the acausal signal of Figure 5.13(c).

Although the short term EGFA envelope rarely yields the modelled inter-hydrophone travel time based on the measured sound speed profile throughout the 84 hour period, the EGFA envelopes of the summed cross- 
correlations for this period do peak at times near the simulated travel times, as shown at the far right of Figure 5.13(a)-(c). The surface reflected path is particularly strong. This is because the cross-correlation is dominated by nearby ships, and these shorter ranges favour higher grazing angles of the acoustic signals relative to the horizontal.

A strong signal is observed at a cross-correlation time of slightly less than zero for all cross-correlations for August 31 in Figure 5.12(a), suggesting that there is a high amplitude signal from near-broadside (either SW or NE) of the array during that day. A corresponding peak in the summed SWAMI52 EGFA envelope is seen at $-0.0175 \mathrm{~s}$ at the far right of Figure 5.13(a). The cross-correlations reveal that this peak is a result of signals from 12-14 Z and 18-24 Z on August 31 (see box 'f' and Figure 5.13(f)). The Shark and SWAMI32 EGFA envelopes do not show a strong signal at these times. Hence the dominant signal seen in the SWAMI52 data is likely from a source significantly closer to that array than the others. R/V Oceanus was

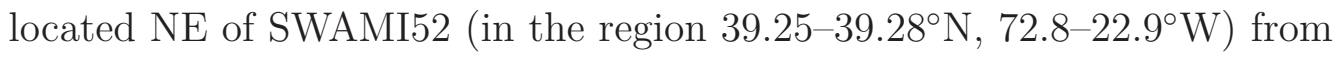
12-24 Z August 31, about $10 \mathrm{~km}$ away. This is the closest that R/V Oceanus came to any of the arrays during the experiment. $\mathrm{R} / \mathrm{V}$ Oceanus moved slowly in the experimental area and as such is an unusual ship noise source. The expected difference in travel time from this near-broadside location to SWAMI52 matches the short time EGFA envelope peaks, as can be seen in Figure 5.13(f). Thus, the high amplitude spurious signals in Figure 5.12(a) are attributed to $\mathrm{R} / \mathrm{V}$ Oceanus. The amplitude of the anomalous $-0.0175 \mathrm{~s}$ peak in the EGFA envelope of the summed normalised cross-correlations, shown in the far right of Figure 5.12(a), decreases to the background noise level when the period 12-24 Z August 31 is excluded, as can be seen in Figure $5.12(\mathrm{~g})$.

Figures 5.12 and 5.13 suggest that the observation time period to obtain a stable EGFA envelope depends on the distribution of the noise. Summing over September 2 yields a good approximation, as shown in Figure 5.13(c), but summing over any of the other days, or even summing over the entire 
84 hour period gives poorer results due to the increased proportion of directional bias of dominant events in the total received signal. Hence, when specific events dominate the cross-correlations, either data from the times during which they occur should be discarded, or the cross-correlations need to be summed over an even longer period so that the effects of individual events are negligible. At any one time, except during the storm, the crosscorrelation is generally dominated by one or two high amplitude events, and eliminating these from the data is difficult. For the case considered here of shipping noise near the coast, the cross-correlations summed over many days or longer could show some directionality, corresponding to preferred shipping routes.

\subsection{Conclusion}

Cross-correlation of ocean noise in the ship dominated $20-100 \mathrm{~Hz}$ frequency band was considered in this chapter. A theoretical stationary-phase based relationship between summed cross-correlations of ship noise using simplifying assumptions was described. The theory showed that the time-derivative of the cross-correlation yields an empirical Green's function approximation (EGFA), an approximation of an amplitude shaded Green's function.

EGFAs were determined from data collected during the Shallow Water 2006 (SW06) experiment. Since ship noise is discrete, long cross-correlation periods were required to give sufficient averaging for the emergence of the Green's function. In this chapter EGFAs were computed over one day, but shorter observation times could potentially be used. The ocean environment is temporally non-stationary. The EGFAs are therefore approximations of 'average', rather than instantaneous, Green's functions. For an appropriate bandwidth, different time and frequency domain normalisation methods yielded similar cross-correlation results. A major reason for this is the spatial averaging of the noise field which occurs when noise from many ship tracks are recorded. 
Direct and surface reflected paths between HLA hydrophones, as well as bottom-surface reflected paths between HLA and VLA hydrophones were determined from the EGFA envelopes for three L-shaped arrays, and agreed well with simulated D, S, and B travel times. Averaging the crosscorrelations between equi-spaced HLA hydrophone pairs increases the signalto-noise ratio.

Analysis of temporal variations in the cross-correlations confirmed that the signal is, at any one time, generally dominated by only one or two sources. Cross-correlations obtained from data recorded during Tropical Storm Ernesto were shown to be clearer than those obtained before and after the storm. This is due to a combination of a reduction in high energy discrete sources (most ships left the area during the storm), and an increase in overall sound levels and hence an increase in signal-to-noise ratio. The source of a dominant spurious signal that is observed in the data on two separate non-consecutive days was identified, and its removal was shown to improve the EGFA.

The results obtained here for ambient noise cross-correlation will be compared with those from active source cross-correlation in Chapter 6 . The arrival times, but not the amplitudes, of the direct and many of the surface-reflected paths were, overall, well estimated using ambient noise cross-correlation of data recorded during Tropical Storm Ernesto. Two practical applications for the direct path arrival time estimates from the SWAMI32 EGFA data will be detailed in Chapter 7, that is, monitoring and localisation of array elements.

If sub-bottom arrival times could also be estimated then ambient noise cross-correlation could potentially be used to estimate sediment properties such as layer depth and sound speed. One aspect of the future work outlined in Section 8.2.5 discusses how low frequency ambient noise data that will be collected on a recently deployed gas hydrate sea-floor observatory will be cross-correlated in an attempt to extract sub-bottom paths and subsequently monitor a gas hydrate mound. 